\title{
A countable dense homogeneous set of reals of size $\aleph_{1}$
}

\author{
by \\ Ilijas Farah (Toronto and Beograd), Michael Hrušák (México) and \\ Carlos Azarel Martínez Ranero (México)
}

\begin{abstract}
We prove there is a countable dense homogeneous subspace of $\mathbb{R}$ of size $\aleph_{1}$. The proof involves an absoluteness argument using an extension of the $L_{\omega_{1} \omega}(Q)$ logic obtained by adding predicates for Borel sets.
\end{abstract}

A separable topological space $X$ is countable dense homogeneous (CDH) if, given any two countable dense subsets $D$ and $D^{\prime}$ of $X$, there is a homeomorphism $h$ of $X$ such that $h[D]=D^{\prime}$. The main purpose of this note is to show the following.

THEOREM 1. There is a countable dense homogeneous set of reals $X$ of size $\aleph_{1}$. Moreover, $X$ can be chosen to be a $\lambda$-set.

Recall that a set of reals is a $\lambda$-set if all of its countable subsets are relatively $G_{\delta}$. Theorem 1 and the fact that a $\lambda$-set cannot be completely metrizable solve problems 390 and 389 from [5]. A construction of a CDH metric space that is not completely metrizable necessarily uses some form of the Axiom of Choice. In [8] it was shown that under sufficient large cardinal assumptions every $\mathrm{CDH}$ metric space in $L(\mathbb{R})$ is completely metrizable. Our proof of Theorem 1 uses Keisler's completeness theorem for the $L_{\omega_{1} \omega}(Q)$ logic (see $\S 2$ ), and a secondary purpose of this note is in explicitly stating a somewhat general method for proving absoluteness of the existence of an uncountable set of reals whose properties are described using Borel sets as parameters.

2000 Mathematics Subject Classification: 54E52, 54H05, 03E15.

Key words and phrases: countable dense homogeneous, $L_{\omega_{1} \omega}(Q), \lambda$-set.

The first author was partially supported by NSERC. The second author's research was partially supported by grant GAČR 201/03/0933, by a PAPIIT grant IN106705 and CONACYT grant 40057-F. 
1. A meager set. Recall that every compact zero-dimensional subset of $\mathbb{R}$ without isolated points is homeomorphic (even isomorphic as a linearly ordered set) to the Cantor set.

LEMma 1.1. There is an uncountable $F_{\sigma}$-set $F$ containing the rationals $\mathbb{Q}$ as a relative $G_{\delta}$-subset and there is an $F_{\sigma}$-equivalence relation $E$ on $F$ all of whose equivalence classes are countable dense subsets of $\mathbb{R}$ and such that for every dense $A \subseteq \mathbb{Q}$ there is a homeomorphism $h: F \rightarrow F$ satisfying

(1) $h[\mathbb{Q}]=A$,

(2) $h(x) E x$ for every $x \in F$.

Proof. Let $F=\mathbb{Q} \cup D \cup \bigcup_{n \in \mathbb{N}} F_{n}$, where $\mathbb{Q}$ and $D$ are disjoint countable dense subsets of $\mathbb{R}$ and $\left\{F_{n}: n \in \mathbb{N}\right\}$ is a family of pairwise disjoint copies of the Cantor set disjoint from both $\mathbb{Q}$ and $D$ and such that every nonempty open set contains one of the $F_{n}$ s. Obviously, $\mathbb{Q}$ is a relative $G_{\delta}$-subset of $F$. Denote by $\mathcal{C}$ the set of all relatively clopen subsets of all the Cantor sets $F_{n}$. For every pair $U, W \in \mathcal{C}$ fix an increasing homeomorphism $h_{U, W}: U \rightarrow W$. Let $\mathcal{F}$ be the (countable) family of all compositions of finitely many functions of type $h_{U, W}$ and their inverses. Then define $x E y$ if and only if $x, y \in \mathbb{Q} \cup D$ or $y=h(x)$ for some $h \in \mathcal{F}$. The relation $E$ is then obviously an equivalence relation all of whose equivalence classes are countable and dense; being a countable union of compact sets, it is $F_{\sigma}$.

Let $A \subseteq \mathbb{Q}$ be dense. Enumerate $\mathcal{C}$ as $\left\{A_{n}: n \in \mathbb{N}\right\}, \mathbb{Q}$ as $\left\{q_{n}: n \in \mathbb{N}\right\}$, $D$ as $\left\{d_{n}: n \in \mathbb{N}\right\}, D \cup(\mathbb{Q} \backslash A)$ as $\left\{c_{n}: n \in \mathbb{N}\right\}$ and $A$ as $\left\{a_{n}: n \in \mathbb{N}\right\}$. Using the back-and-forth argument of Cantor, construct the homeomorphism $h$ : $F \rightarrow F$ as an increasing union of strictly increasing partial homeomorphisms $h_{n}$ so that, for every $n \in \mathbb{N}$ :

(1) $h_{n}$ extends $h_{n-1}$,

(2) $\operatorname{dom}\left(h_{n}\right)$ consists of a finite subset of $\mathbb{Q} \cup D$ and a finite union of elements of $\mathcal{C}$

(3) range $\left(h_{n}\right)$ consists of a finite subset of $\mathbb{Q} \cup D$ and a finite union of elements of $\mathcal{C}$,

(4) $h_{n}$ restricted to $\operatorname{dom}\left(h_{n}\right) \backslash(\mathbb{Q} \cup D)$ is covered by finitely many elements of $\mathcal{F}$,

(5) $h_{n}(q) \in A$ for every $q \in \mathbb{Q} \cap \operatorname{dom}\left(h_{n}\right)$,

(6) $h_{n}(d) \in D \cup(\mathbb{Q} \backslash A)$ for every $d \in D \cap \operatorname{dom}\left(h_{n}\right)$,

(7) $\left\{q_{m}: m \leq n\right\} \cup\left\{d_{m}: m \leq n\right\} \cup \bigcup\left\{A_{m}: m \leq n\right\} \subseteq \operatorname{dom}\left(h_{n}\right)$,

(8) $\left\{a_{m}: m \leq n\right\} \cup\left\{c_{m}: m \leq n\right\} \cup \bigcup\left\{A_{m}: m \leq n\right\} \subseteq \operatorname{range}\left(h_{n}\right)$.

Then $h=\bigcup_{n \in \mathbb{N}} h_{n}$ is the desired homeomorphism of $F$. It maps $\mathbb{Q}$ onto $A$ by (5), (7) and (8). It is a bijection by (7) and (8) and both $h$ and $h^{-1}$ are continuous as they are strictly increasing mappings of a dense set onto a dense set $(\mathbb{Q}$ onto $A$ and vice versa). 
Recall that if $E$ is an equivalence relation then a set $X$ is $E$-saturated if for all $x E y$ we have $x \in X$ if and only if $y \in X$.

Lemma 1.2. Assume $\mathbb{Q}, D, F, E$ and $\mathcal{F}$ are as in Lemma 1.1 and its proof. If $X \subseteq F$ is an $E$-saturated set such that for every countable $B \subseteq X$ there is an E-saturated $A \subseteq X$ containing $B$ and a homeomorphism $h$ : $X \rightarrow X$ satisfying $h[A]=\mathbb{Q}$, then $X$ is countable dense homogeneous.

Proof. Fix a countable dense subset $B$ of $X$. Let $g$ be an autohomeomorphism of $X$ such that $g^{-1}(\mathbb{Q})$ is an $E$-saturated set containing $B$. Then $A=g[B]$ is a dense subset of $\mathbb{Q}$. By Lemma 1.1 there is an autohomeomorphism $h$ of $F$ such that $h[\mathbb{Q}]=A$ and $h(x) E x$ for every $x \in F$. Therefore $h \uparrow X$ is an autohomeomorphism of $X$. Then $H=h^{-1} \circ g$ is an autohomeomorphism of $X$ such that $H[B]=\mathbb{Q}$ as required.

2. Absoluteness. The $L_{\omega_{1} \omega}(Q)$ logic is an extension of first-order logic obtained by adding the following two syntactical rules:

(1) If $\phi_{n}(n \in \mathbb{N})$ are formulas and the set of all variables that are free in some of these formulas is finite, then $\bigwedge_{n} \phi_{n}$ is a formula.

(2) If $\phi$ is a formula, then so is $(Q x) \phi$.

Given a structure $\mathfrak{X}$ of the appropriate signature, the satisfaction relation $\mathfrak{X} \models \phi$ is defined by recursion on the complexity of $\phi$. The new syntactic constructions are interpreted in the intuitive way. First, $\mathfrak{X} \models \bigwedge_{n} \phi_{n}(a)$ if and only if $\mathfrak{X} \models \phi_{n}(a)$ for all $n$. The intended interpretation for $Q x$ is 'there exist uncountably many', and we define $\mathfrak{X} \models Q x \phi(x, a)$ if and only if the set of $b \in X$ such that $\mathfrak{X} \models \phi(b, a)$ is uncountable. (Here $X$ denotes the universe of $\mathfrak{X}$, and $a$ is an element of $X$.) When $Q x$ is defined (interpreted) in this way we say that a model is standard.

The expressive power of $L_{\omega_{1} \omega}(Q)$ is a bit higher than that of the firstorder logic. Nevertheless, the completeness of $L_{\omega_{1} \omega}(Q)$ with respect to a finite set of axioms and rules of inference was proved by Keisler ([10]): A sentence $\phi$ has a standard model if and only if there is no proof for $\neg \phi$. Proofs in $L_{\omega_{1} \omega}(Q)$ can be coded by well-founded countable trees, and therefore the statement 'there is no proof for $\phi$ ' is absolute between models of ZFC. Keisler's theorem is therefore useful for proving absoluteness of the existence of certain objects of size $\aleph_{1}$ (see $\left.[9,1,3,6,7,11]\right)$.

Let $L_{\omega_{1} \omega}^{\mathrm{B}}(Q)$ be the extension of $L_{\omega_{1} \omega}(Q)$ allowing countably many Borel predicates in the following sense. For a Borel set $A \subseteq(\mathbb{R})^{k(A)}$, a Borel function $f:(\mathbb{R})^{l(f)} \rightarrow \mathbb{R}(k(A) \in \mathbb{N}, l(f) \in \mathbb{N})$ and a real $b \in \mathbb{R}$ we have relation, function, and constant symbols $\mathbf{A}, \mathbf{f}$ and $\mathbf{b}$, respectively, where $\mathbf{A}$ and $\mathbf{f}$ are of matching arity. We also add a unary predicate symbol $\mathbf{R}$. If $\phi$ is a sentence of $L_{\omega_{1} \omega}^{\mathrm{B}}(Q)$ we say that a model $\mathfrak{X}$ of $\phi$ (with universe $X$ ) is 
correct if

(1) $\mathbf{R}$ is interpreted as $\mathbb{R} \cap X$,

(2) $\mathbf{A}, \mathbf{f}$ and $\mathbf{b}$ occurring in $\phi$ are interpreted as $A \cap X^{k(A)}, f\left\lceil X^{l(f)}\right.$ and $b$, respectively, and

(3) if A occurring in $\phi$ is countable, then $A \subseteq X$.

THeOREM 2. An $L_{\omega_{1} \omega}^{\mathrm{B}}(Q)$-sentence $\phi$ has a correct model whenever it has a correct model in some forcing extension $V^{\mathbb{P}}$ of the universe $V$.

More is true: An $L_{\omega_{1} \omega}^{\mathrm{B}}(Q)$-sentence $\phi$ has a correct model if and only if it has a correct model in some forcing extension $V^{\mathbb{P}}$ of the universe $V$. See Remark 2.2.

Let us postpone the proof of Theorem 2 for a moment. Fix an $L_{\omega_{1} \omega}^{\mathrm{B}}(Q)$ sentence $\phi$. We shall associate an $L_{\omega_{1} \omega}(Q)$-sentence $\phi^{M}$ to $\phi$. Let us treat the case when $\phi$ involves a predicate for only one Borel set, $A \subseteq \mathbb{R}$. Via a standard coding argument this extends to cover the general case. First, the language of $\phi$ is expanded by adding new symbols $\mathbf{Q},\left\{\mathbf{c}_{q}: q \in \mathbb{Q}\right\}$ and $<$. Let $\psi_{0}$ be the conjunction of sentences stating the following:

(1) $(\forall x)\left(\mathbf{Q}(x) \Leftrightarrow \bigvee_{q \in \mathbb{Q}} x=\mathbf{c}_{q}\right)$,

(2) $\bigwedge_{p<q} \mathbf{c}_{p}<\mathbf{c}_{q}$,

(3) $\bigwedge_{p \in \mathbb{Q}} \mathbf{R}\left(\mathbf{c}_{p}\right)$,

(4) $<$ is a linear ordering of (the interpretation of) $\mathbf{R}$,

(5) $\mathbf{R}(x) \wedge \mathbf{R}(y) \Rightarrow(\exists z)(\mathbf{Q}(z) \wedge \neg(x<z \Leftrightarrow y<z))$.

(The last clause states that the rationals are dense in $<$.) We can now define a formula $\psi^{A}(x)$ by recursion on the Borel complexity of $A$. If $A$ is an open set, then it can be written as a union of rational intervals $\bigcup_{n}(p(n), q(n))$, and we let $\psi^{A}(x)$ be $\bigvee_{n}\left(\mathbf{c}_{p(n)}<x \wedge x<\mathbf{c}_{q(n)}\right)$. If $\psi^{A}$ has been defined, let $\psi^{\mathbb{R} \backslash A}(x)$ be $\neg \psi^{A}(x)$. If $\psi^{A_{n}}$ has been defined, let $\psi^{\bigcap_{n} A_{n}}(x)$ be $\bigwedge_{n} \psi^{A_{n}}(x)$. Let $\phi_{0}^{M}$ be obtained from $\phi^{M}$ by replacing each occurrence of $\mathbf{A}$ with $\psi^{A}$, and finally let $\phi^{M}$ be $\phi_{0}^{M} \wedge \psi_{0}$.

The following is now obvious.

Lemma 2.1. An $L_{\omega_{1} \omega}^{\mathrm{B}}(Q)$-sentence $\phi$ has a correct model if and only if $\phi^{M}$ has a standard model.

Proof of Theorem 2. By Lemma 2.1, $\phi$ has a correct model if and only if $\phi^{M}$ has a standard model. By Keisler's completeness theorem for $L_{\omega_{1} \omega}(Q)$ ([10]), $\phi^{M}$ has a standard model if and only if there is no proof for $\neg \phi^{M}$. However, a proof for $\neg \phi^{M}$, being a well-founded tree, remains a proof in the extension.

REMARK 2.2. To see that the converse of Theorem 2 holds, note that if $\phi^{M}$ has a model $\mathfrak{X}$ in $V$ then $\mathfrak{X}$ is a weak model (see [10]) of $\phi^{M}$ in $V^{\mathbb{P}}$, and again by Keisler's theorem $\phi^{M}$ has a standard model in $V^{\mathbb{P}}$. 
In the following lemma, $\mathbf{A}, \mathbf{B}, \mathbf{C}$ and $\mathbf{D}$ are unary relation symbols, $\mathbf{h}$ is a unary function symbol and $\mathbf{f}$ is a binary function symbol. We say that a property is expressible in $L_{\omega_{1} \omega}^{\mathrm{B}}(Q)$ if there is a sentence of $L_{\omega_{1} \omega}^{\mathrm{B}}(Q)$ such that in each of its correct models the interpretations $A, B, C, D, f, h$ of these predicates satisfy the stated property. A linear order is $\omega_{1}$-like if it is uncountable while each of its initial segments is countable.

Lemma 2.3. The following properties are expressible in $L_{\omega_{1} \omega}^{\mathrm{B}}(Q)$.

(1) $A$ is countable.

(2) A binary relation $<$ is an $\omega_{1}$-like linear order.

(3) $h: A \rightarrow B$ is a surjection.

(4) $h: A \rightarrow B$ is a continuous function.

(5) $h: A \rightarrow B$ is a homeomorphism.

(6) $h: A \rightarrow B$ and $h[C]=D$.

(7) $f(x, \cdot): A \rightarrow B$ is a homeomorphism for every $x$.

(8) $x$ is in the closure of $A$.

(9) $A$ is a dense subset of $B$.

(10) $A$ is a relatively open subset of $B$.

(11) $A$ is a relatively $G_{\delta}$ subset of $B$.

(12) $B$ has a countable dense subset $K$ that is relatively $G_{\delta}$ in $B$.

(13) $X$ is E-saturated, for a given Borel equivalence relation $E$ all of whose equivalence classes are countable.

Proof. Items (3) and (6) are first-order definable, and (1) and (2) are straightforward to define using $Q x$.

For (4), (5) and (8) one only needs to observe that since we have a standard model of $L_{\omega_{1} \omega}(Q)$, quantifiers such as $(\forall \varepsilon>0)(\exists \delta>0)$ are evaluated correctly. Item (7) is immediate from the preceding items, and (10) and (9) are immediate from (8). For (11), introduce new predicates $\mathbf{A}_{n}(n \in \mathbb{N})$ and require that each $A_{n}$ is a relatively open subset of $B$ and $A=\bigcap_{n} A_{n}$.

To see (12), add a predicate for $A$ and then use (1), (2), (9) and (11).

Let $E$ be as in (13). It is well known that there are Borel functions $f_{n}$ $(n \in \mathbb{N})$ such that $x E y$ if and only if $(\exists n) x=f_{n}(y)$, hence for (13) we only need to add names for $f_{n}(n \in \mathbb{N})$ to our language.

It turns out that one cannot use Keisler's theorem to extend $L_{\omega_{1} \omega}^{\mathrm{B}}(Q)$ to include analytic sets as predicates. Moreover, obtaining absoluteness of existential sentences involving the quantifier $Q x$ and predicates for analytic sets is not possible by using any means unless some large cardinal asssumption is made. On the other hand, if there are class many Woodin cardinals then the analogue of Theorem 2 holds not only for analytic sets, but even when arbitrary universally Baire predicates are allowed (see [4]). 
3. Proof of Theorem 1. Assume $\mathbb{Q}, D, F, E$ and $\mathcal{F}=\left\{g_{n}: n \in \mathbb{N}\right\}$ are as in Lemma 1.1 and its proof. By Lemma 1.2, an uncountable $E$-saturated $X \subseteq F$ with an $\omega_{1}$-like ordering $<$ such that

(1) each $E$-equivalence class is an interval in $<$,

(2) there is a function $H: X \times X \rightarrow X$ such that for every $x \in X$ :

(a) $H(x, \cdot)$ is an autohomeomorphism of $X$,

(b) $H(x, y) \in \mathbb{Q}$ if and only if $y<x$

will be countable dense homogeneous. By Lemma 2.3, the existence of $X$ and $H$ can be expressed in $L_{\omega_{1} \omega}^{\mathrm{B}}(Q)$, and by Theorem 2 it suffices to show that $X$ exists in some forcing extension. In order to ensure that $X$ is uncountable, we will force with a ccc poset. In [2] it was proved that if $\left\{C_{\alpha}: \alpha<\omega_{1}\right\}$ and $\left\{D_{\alpha}: \alpha<\omega_{1}\right\}$ are two families of pairwise disjoint countable dense subsets of $\mathbb{R}$ then some ccc forcing adds a homeomorphism $h: \bigcup_{\alpha<\omega_{1}} C_{\alpha} \rightarrow \bigcup_{\alpha<\omega_{1}} D_{\alpha}$ such that $h\left[C_{\alpha}\right]=D_{\alpha}$ for every $\alpha<\omega_{1}$. Therefore, if we pick any $\omega_{1}$-sequence of equivalence classes so that the first one is $\mathbb{Q} \cup D$ and let $X$ be their union then a standard ccc forcing such that $\mathrm{MA}_{\aleph_{1}}$ holds in the extension adds $H$ with the required properties.

Since $\mathbb{Q}$ is a relatively $G_{\delta}$-subset of $F$, it is a countable dense and relatively $G_{\delta}$-subset of $X$. By countable dense homogeneity, $X$ is a $\lambda$-set.

\section{References}

[1] J. Bagaria, Fragments of Martin's axiom and $\boldsymbol{\Delta}_{3}^{1}$ sets of reals, Ann. Pure Appl. Logic 69 (1994), 1-25.

[2] S. Baldwin and R. E. Beaudoin, Countable dense homogeneous spaces under Martin's axiom, Israel J. Math. 65 (1989), 153-164.

[3] I. Farah, A coherent family of partial functions on $\mathbb{N}$, Proc. Amer. Math. Soc. 124 (1996), 2845-2852.

[4] I. Farah and P. Larson, Absoluteness for universally Baire sets and the uncountable, in preparation, 2005.

[5] B. Fitzpatrick Jr. and H.-X. Zhou, Some open problems in densely homogeneous spaces, in: J. van Mill and M. Reed (eds.), Open Problems in Topology, NorthHolland, Amsterdam, 1984, 251-259.

[6] J. Hirschorn, Summable gaps, Ann. Pure Appl. Logic 120 (2003), 1-63.

[7] - On the strength of Hausdorff's gap condition, preprint, 2004.

[8] M. Hrušák and B. Zamora Avilés, Countable dense homogeneity of definable spaces, Proc. Amer. Math. Soc., to appear.

[9] J. I. Ihoda and S. Shelah, Souslin forcing, J. Symbolic Logic 53 (1988), 1188-1207.

[10] H. J. Keisler, Logic with the quantifier "there exist uncountably many", Ann. Math. Logic 1 (1970), 1-93. 
[11] W. Kubiś and S. Shelah, Analytic colorings, Ann. Pure Appl. Logic 121 (2003), 145-161.

Department of Mathematics and Statistics

Instituto de Matemáticas

York University

UNAM

4700 Keele Street

Unidad Morelia, A.P. 61-3

Toronto, Canada M3J 1P3

Xangari, C.P. 58089

E-mail: ifarah@mathstat.yorku.ca

Morelia, Mich., México

Web: http://www.mathstat.yorku.ca/ ifarah

E-mail: michael@matmor.unam.mx

azarel@matmor.unam.mx

Matematicki Institut

Kneza Mihaila 35

11000 Beograd

Serbia and Montenegro

Received 12 November 2004;

in revised form 2 June 2005 